\title{
A New Quality Control Technology Based on Relay Chain Theory
}

\author{
Bo Wang ${ }^{1, *}$, Zhi-yong $\mathrm{Li}^{1}$, Hou-gui Zhou ${ }^{2}$ and Xiang-tian Nie ${ }^{1}$ \\ ${ }^{I}$ College of Water Conservancy, North China University of Water Resources and Electric Power, Zhengzhou, China \\ ${ }^{2}$ China Energy Engineering Group Co., Ltd, Beijing, China
}

\begin{abstract}
Activity handover within engineering construction projects is time consuming, takes longer than the actual activities, and therefore, can cause duration delays. This paper presents relay chain seamless handover (RCSH) technology, which is based on relay technology theory and actor-network theory. This study systematically elaborated the RCSH processes, activity relay flows, and methods to address balancing speed under varying conditions. Our analysis results indicate that as compared to traditional activity handover, RCSH improves handover quality and guarantees the duration. RCSH technology was successfully applied in the third phase of the Three Gorges Dam Project, specifically for its concrete construction duration and quality control. Through the use of RCSH, effective overall planning, and efficient coordination of various production links, along with seamless activity handover in the third phase of the project were accomplished — with no additional time consumed by the handover process - and the construction of a concrete dam without a crack was achieved.
\end{abstract}

Keywords: Actor-network theory, balancing speed, relay chain, seamless handover, three gorges dam project.

\section{INTRODUCTION}

Typical program management methods such as Gantt chart $[1,2]$, network planning $[3,4]$, and the newly proposed visualization of construction schedules $[5,6]$ often do not account for the time required for activity handover. For actual engineering construction projects, activity handover can be time consuming, at times takes longer than the actual activities, and can therefore cause duration delays. High quality requirements, difficult construction, and concreteness in hot seasons characterized concrete construction of the third phase of the Three Gorges Dam Project power plant section. More than 3,000 concrete storehouses were completed in this project. Each completed storehouse underwent multiple activities involving numerous departments and operation teams. How construction workers planned such a large production system as a whole and efficiently coordinated numerous, disparate production activities to achieve seamless handover are important subjects to be studied.

In the 1980s, French sociologists Bruno Latour, Michel Callon, and Jones Law developed the actor-network theory (ANT) [7-9], in which they redefined the roles of various existences (science, technology, nature, society, spirit, knowledge, etc.) in the process of scientific understanding, and used actors to represent these existences. Each actor in the actor-network is a node and the actors interconnected form a scientific knowledge network. In this network, the statuses of actors are equal: there is no so-called network center and no subject-object relationships; each node is an independent "actor" in participating scientific knowledge

*Address correspondence to this author at the College of Water Conservancy, North China University of Water Resources and Electric Power, Zhengzhou, China; E-mail: wangbosky99@163.com
$[10,11]$. Translation, in which actors constantly strive to convert other actors' issues and interests in their own language, is the basic method and tool for communication within the actor-network [12]. When constructing a scientific actornetwork, constructors themselves and the translated actors together build a seamless web of action through a variety of translation methods or patterns. Each actor has its own interests and can persuade other actors to resonate with them on these interests to form an alliance [13, 14]. Chan illustrated that there are also not only human but non-human influencing factors in the success of the construction project [15]. Study of the actor-network suggests that engineering activities, with both technical and non-technical content [17], are the situational practices that combine together to form a conceptual and political power network $[16,18]$. Engineering activity is an integrated process of heterogeneous elements which are not given and freely available for innovators. Only those elements that have been identified, perceived, transferred, and translated into the actor-network can play a role. In engineering practice, activities are the core actors in the actor-network and form a seamless web of action through translation processes. Therefore, for constructing the engineering actor-network, it is necessary to study how to implement efficient handover or translation among activities to achieve seamless handover.

The relay operation is a method for studying how to automatically interconnect the non-linear activities, maintain their operation in an optimum state, and create and protect the corresponding external environment. The method emphasizes the perspective that "in-activity (introverted activity, pointed to the relay event) and ex-activity (extroverted activity, emitted from the relay event) are each other's service." This breaks from the ideological limitations of total quality management, which emphasizes that the "next activity is customer" and "customer is God." It views a specific activity 
as a "basic element" and studies the optimal coordinated action of "receiving" from pre-activity (immediately preceding the activity), "doing" the activity, and "handing" for the post-activity (immediately following the activity). Cauvin presents the concept of actor relay and puts the actor in the heart of the whole disruption management system [19]. All the above-mentioned research viewed activity as the study object, presented the concept of activities, and emphasized the perspective that "in-activity and ex-activity are each other's service;" however, they did not give an account on the chain or network formed by activity handover, nor did they explain how to implement seamless handover between activities and provide actual quantitative analysis.

This paper presented relay chain seamless handover (RCSH) technology, which is based on the relay technology theory and actor-network theory. As the object of study, $\mathrm{RCSH}$ took the chain or network formed by activity handover; illustrated how to implement efficient handover or translation among activities and systematically elaborated $\mathrm{RCSH}$ processes, activity relay flows, and methods for solving balancing speeds under varying conditions. Comparison and analysis results indicate that RCSH offers advantages over traditional activity handover by improving handover quality and guaranteeing predefined durations.

\section{RELATED WORK}

\subsection{Definitions And Assumptions}

Definition 1: Relay chain refers to the relay network chain comprising all activities, from the initial to the terminant ones. It is formed by operating, collaborating, and exchanging activities in the construction process. Like a link in a chain, each activity is connected to form an interrelated whole.

Relay chain consists of single-relay and multi-relay handover events. The former refers to a single in-activity handover to single ex-activity; the latter refers to a single inactivity handover to multiple ex-activities, or to an exchange between multiple in-activities and multiple ex-activities. The basic principle of activity handover in relay chain technology is similar to the baton exchange principle in a relay race; that is, the baton exchange can have a higher guarantee rate when there is no relative speed between the baton-handing and baton-receiving runners, and the whole race time can be reduced when handover speed increases. In this way, the project quantity can be viewed as a fixed value in which balancing speed decreases when handover speed increases, which thereby improves quality under guaranteed duration.

Definition 2: RCSH technology refers to technology that implements the non-time-consumption of activity handover according to the relay technology principle and activity handover methods. It emphasizes synchronized operations among various departments and the project overall, adheres to punctuality, strives for advance docking, and implements activity balancing construction using a reserve of resources and resulting in a release for uncontrolled states.

The seamless handover principle for activities centers on the overall goa, in which each activity relates to the whole process of "receiving, doing, and handing." The principle therefore adheres to pre-activity as being its "own business," entering a competitive state in advance, while also adhering to post-activity as being "God." In this way, the "default" of the pre-activity is fully satisfied and superior conditions are "inter-created" for pre-activity and post-activity by completing the given task qualitatively and efficiently. Thus, the activity can be completed on time or in advance.

In this paper, RCSH refers to the dynamic relay chain for seamless handover. For comparison, static single relay chain for seamless handover, which is the special RCSH condition when its handover speed is zero, is outlined in Section 2.3.2.

Definition 3: Balancing speed refers to accounting for the degree of difficulty of (engineering) activity, equipment configuration utilization ratio, and resource reserve. Each weight in order to be balanced completes the amount of engineering quantities on average within a day. The balancing speed calculation formula is as follows:

$$
\begin{aligned}
& V=\frac{A}{T \sum_{j=1}^{5} Q_{i j} X_{i j} P S / \lambda B} \\
& \quad \text { Let } \gamma=\sum_{j=1}^{5} Q_{i j} X_{i j} P S / \lambda B, \text { that } \mathrm{V}=\mathrm{A} / \mathrm{T} \lambda .
\end{aligned}
$$

It is assumed that for any relay event in a relay chain network-planning diagram, the input is "and" type. That is, if and only if all in-activities are realized, the input can be implemented; the implementation time is the maximum of all in-activity implementation times. The output is the affirmation type; that is, if the relay event is implemented, all exactivities must be realized, and the implementation probability value of each ex-activity is equal to 1 .

It is also assumed that in the relay chain network, the speed of all activity acceleration processes - from the beginning of construction (the starting or handover speed) to the balancing speed, or from the balancing speed to the construction end (the handover speed or termination) - are uniform.

\subsection{Definitions Of Symbols}

$\mathbf{i}, \mathbf{j}, \mathbf{k}$ : Activities interconnected as a sequence

A: Activity engineering quantities (work day)

T: Activity planned duration (work day)

1/B: Activity difficulty (very difficult, 0.2 ; difficult, 0.4 ; medium, 0.6; easy, 0.8; very easy, 1)

Qij: The ith activity and jth title weight: 1) senior engineer with professorship, 9; 2) senior engineer, 7; 3) engineer, 5; 4) assistant engineer, 3; 5) mechanic, 1

Xij: Number of people of the ith activity with jth title

P: Equipment configuration, according to the difference in quality, respectively referred as $1,0.8,0.6$, and 0.4.

S: Equipment utilization ratio, according to the level of utilization, respectively referred as $1,0.8,0.6$, and 0.4 . 


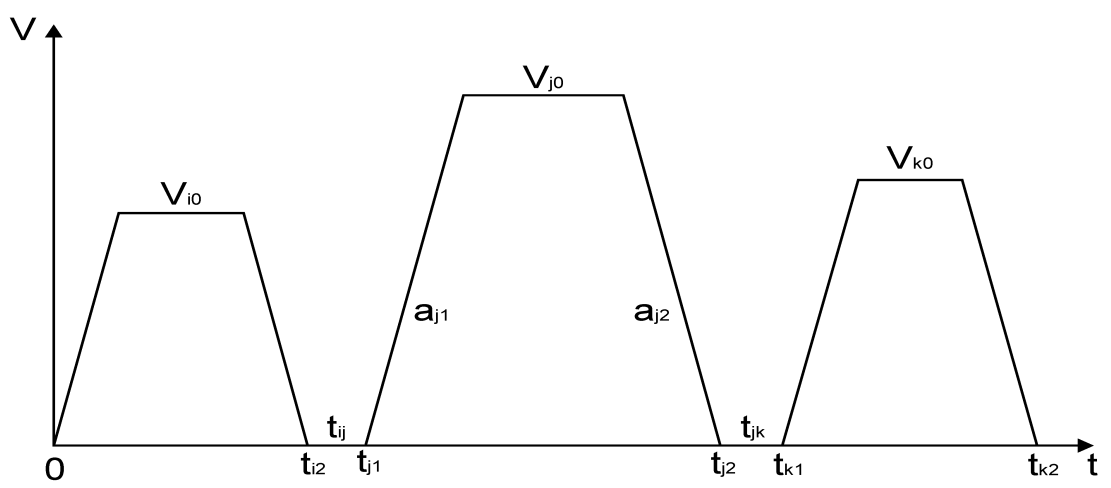

Fig. (1). Schematic diagram of traditional handover between single activities.

$\lambda$ : Resources reserve coefficient. The guarantee system of relay chain network operation mainly depends on the procession of various operating conditions, including personnel, materials, labor, equipment, capital, technology, etc. According to activity-specific characteristics, the reserve coefficient should reach the theoretical value $\lambda(\lambda \geq 1)$ times; for some complex or particularly important construction activities, the reserve coefficient will be higher.

ti1, tj1, tk1: Starting time of activity $\mathrm{i}, \mathrm{j}, \mathrm{k}$

ti2, tj2, tk2: Finishing time of activity $i, j, k$

tij, tjk: Handover time of activity $i, j, k, t i j=t j 1-t i 2$, $\mathrm{tjk}=\mathrm{tk} 1-\mathrm{tj} 2$

ai1, aj1, ak1: Acceleration of activity i, j, k from starting time (speed is 0 ) or handover speed to balancing speed

ai2, aj2, ak2: Acceleration of activity i, $\mathrm{j}, \mathrm{k}$ from balancing speed to handover speed or finish time (speed is 0 )

vi0, vj0, vk0: Balancing speed of activity i, j, k in traditional handover

vi1, vj1, vk1: Balancing speed of activity $i, j, k$ in singleRCSH (static)

vi2, vj2, vk2: Balancing speed of activity i, $\mathrm{j}, \mathrm{k}$ in single$\mathrm{RCSH}$

$\boldsymbol{\mu}$ : Calculation speed of activity handover, which takes the minimum balancing speed among in-activities and exactivities

$\boldsymbol{\beta}:$ Handover speed conversion coefficient, $\beta=0.85 \sim 0.95$

$\omega$ : Activity handover speed, $\omega=\beta \mu$

rij, rjk: Handover buffer time of activity $i, j$ and $j, k$, rij, rjk $=0.1 \sim 0.3$

zij, zjk: Handover preparation time of activity $\mathbf{i}, \mathbf{j}$, and $\mathrm{j}$, k. In RCSH technology, the in-activity and ex-activity should overlap; the ex-activity should take the initiative to receive the in-activity and be prepared in advance, just as a baton-receiving runner in a relay race starts in advance and accelerates to handover speed. Generally, zij, zjk $=0.2 \sim 0.4$, z $=0.25$ in the third phase of the Three Gorges Dam Project.

\subsection{Single-RCSH}

\subsubsection{Traditional Handover Between Single Activities}

There is no relay in traditional activity handover; it was only a simple handover process. The handover is time consuming and does not produce any effective quantities in the handover time. An ex-activity must start from zero speed after receiving the "baton," as it were, so that the balancing speed will be high, which thereby increases the duration delay risk. Schematic diagram of traditional handover between single activities is shown in Fig. (1).

Traditional activity balancing speed vj0 solutions met the following equation:

$$
\begin{gathered}
\frac{v_{j 0}^{2}}{2 a_{j 1}}+\frac{v_{j 0}^{2}}{2\left|a_{j 2}\right|}+\left(T_{j}-t_{i j}-\frac{v_{j 0}}{a_{j 1}}-\frac{v_{j 0}}{\left|a_{j 2}\right|}\right) v_{j 0}=\frac{A_{j}}{\lambda_{j}} \\
\text { For } v_{j 0}=\frac{\left(T_{j}-t_{i j}\right)+\sqrt{\left(T_{j}-t_{i j}\right)^{2}-\left(\frac{1}{a_{j 1}}+\frac{1}{\left|a_{j 2}\right|}\right) \frac{2 A_{j}}{\lambda_{j}}}}{1 / a_{j 1}+1 /\left|a_{j 2}\right|} \\
>\frac{T_{j}-t_{i j}}{1 / a_{j 1}+1 /\left|a_{j 2}\right|}=\frac{a_{j 1}\left|a_{j 2}\right|}{a_{j 1}+\left|a_{j 2}\right|}\left(T_{j}-t_{i j}\right)=v_{j 0 \max }
\end{gathered}
$$

With discrepancy and dropping, the following demonstration was the same. Hence:

$$
v_{j 0}=\frac{\left(T_{j}-t_{i j}\right)-\sqrt{\left(T_{j}-t_{i j}\right)^{2}-\left(\frac{1}{a_{j 1}}+\frac{1}{\left|a_{j 2}\right|}\right) \frac{2 A_{j}}{\lambda_{j}}}}{1 / a_{j 1}+1 /\left|a_{j 2}\right|}
$$

\subsubsection{Single-RCSH (Static)}

Again using the relay-race metaphor, seamless and static handover refers to the baton-receiving runner standing still, however, waiting to receive the baton. Although, this receiving method is safe and the baton exchange does not consume time, the baton-handing runner must slow down considerably to pass (and not drop) the baton, while the baton-receiving runner must start from zero speed and then accelerate. Seamless static handover involves an analogous activity relay, in 


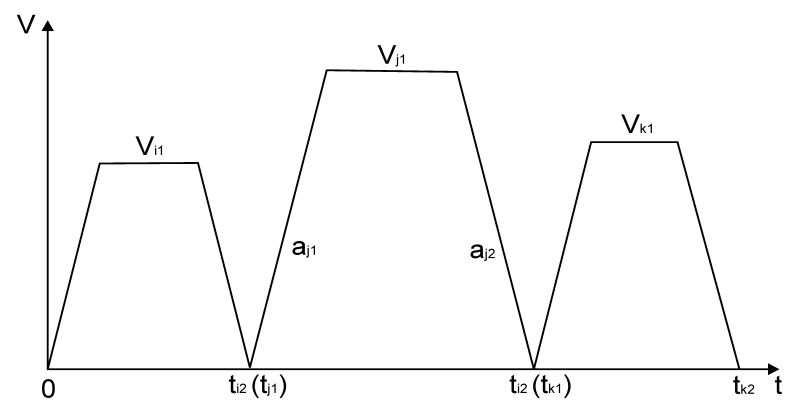

Fig. (2). Schematic diagram of single-RCSH (Static).

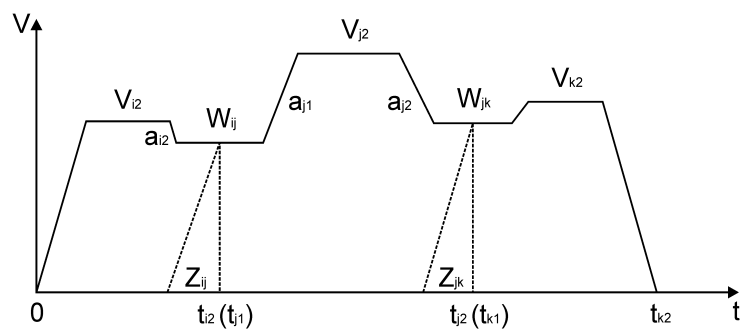

Fig. (3). Schematic diagram of single-RCSH.

which the relay process of the in-activity "hands over," and the ex-activity "receives." Schematic diagram of singleRCSH (static) is shown in Fig. (2).

Let $t_{i j}=0$ in formula (3), in single-RCSH (static), the equation for the balancing speed $v_{j l}$ of $j$ activity is:

$v_{j 1}=\frac{T_{j}-\sqrt{T_{j}^{2}-\left(\frac{1}{a_{j 1}}+\frac{1}{\left|a_{j 2}\right|}\right) \frac{2 A_{j}}{\lambda_{j}}}}{1 / a_{j 1}+1 /\left|a_{j 2}\right|}$

\subsubsection{Single- $\mathrm{RCSH}$}

An object has the characteristic of inertia for maintaining a state; it must therefore constantly accelerate for some time to reach a certain speed. Using this law, to reduce the time consumed during acceleration in a relay race, the batonpassing runner slows down before the baton exchange, while the baton-receiving runner accelerates before the exchange and receives the baton when accelerating to a certain speed. The receiver then maintains the best running speed until the baton is handed to the next baton-receiving runner.

$\mathrm{RCSH}$ is analogous to the above process. RCSH activity handover does not consume time, and the balancing speed of each activity is relevant to the balancing speeds of the preactivity and post-activity. For example, a baton-passing runner does not need to pass the baton at zero speed, nor does the baton-receiving runner need to begin the handover process and accelerate from zero speed. To ensure a smooth handover between in-activity and ex-activity in RCSH, some handover speed is sacrificed (the handover speed that is less than the balancing speed). The in-activity speed slows to the handover speed, and the ex-activity accelerates to the handover speed through advance preparation (although actual construction does not yet begin, it can be started from this speed). Subsequently, the ex-activity accelerates to balancing speed after the handover. This handover process has the advantages of high stability and low handover failure probability. Schematic diagram of single-RCSH is shown in Fig. (3) and single-RCSH flow chart is shown in Fig. (4).

The balancing speed vi2, vj2, vk2 of activity $i, j, k$ respectively, satisfies equation (5), (6), (7):

$$
\begin{aligned}
& \frac{v_{i 2}^{2}}{2 \mathrm{a}_{i 1}}+\frac{1}{2} r_{i j} \omega_{i j}+\frac{v_{i 2}^{2}-\omega_{i j}^{2}}{2\left|a_{i 2}\right|}+\left(T_{i}-\frac{v_{i 2}}{a_{i 1}}-\frac{v_{i 2}-\omega_{i j}}{\left|a_{i 2}\right|}-\frac{1}{2} r_{i j}\right) v_{i 2}=\frac{A_{i}}{\gamma_{i}}(5) \\
& \frac{1}{2} r_{i j} \omega_{\mathrm{ij}}+\frac{v_{j 2}^{2}-\omega_{i j}^{2}}{2 a_{j 1}}+\frac{1}{2} r_{j k} \omega_{j k}+\frac{v_{j 2}^{2}-\omega_{j k}^{2}}{2\left|a_{j 2}\right|} \\
& +\left(T_{j}-\frac{1}{2} r_{i j}-\frac{1}{2} r_{j k}-\frac{v_{j 2}-\omega_{i j}}{a_{j 1}}-\frac{v_{j 2}-\omega_{j k}}{\left|a_{j 2}\right|}\right) v_{j 2}=\frac{A_{j}}{\gamma_{j}} \\
& \frac{1}{2} r_{j k} \omega_{j k}+\frac{v_{k 2}^{2}-\omega_{j k}^{2}}{2 a_{k 1}}+\frac{1}{2} r_{k l} \omega_{k l}+\frac{v_{k 2}^{2}-\omega_{k l}^{2}}{2\left|a_{k 2}\right|}+\left(T_{k}-\frac{1}{2} r_{j k}\right. \\
& \left.-\frac{1}{2} r_{k l}-\frac{v_{k 2}-\omega_{j k}}{a_{k 1}}-\frac{v_{k 2}-w_{k l}}{\left|a_{k 2}\right|}\right) v_{k 2}=\frac{A_{k}}{\gamma_{k}}
\end{aligned}
$$

In the balancing-speed solving process, two balancing speeds can be selected by the handover calculation speed $\mu$ on each relay event. If there are $n$ relay events, $2 \mathrm{n}$ balancing speeds can be chosen. Let $\mathrm{M}=\{\mathrm{d} 1=\{\mu 1 \mathrm{ij}, \quad \mu 1 \mathrm{jk}, \ldots$, $\mu 1 \mathrm{mn}\}, \mathrm{d} 2=\{\mu 2 \mathrm{ij}, \mu 2 \mathrm{jk}, \ldots, \mu 2 \mathrm{mn}\}, \ldots, \mathrm{d} 2 \mathrm{n}=\{\mu 2 \mathrm{nij}$, $\mu 2 \mathrm{njk}, \ldots, \mu 2 \mathrm{nmn}\}\}$. To obtain each activitys' balancing speed, the element $\mathrm{d}$ of set $\mathrm{M}$ is sequentially selected, the 


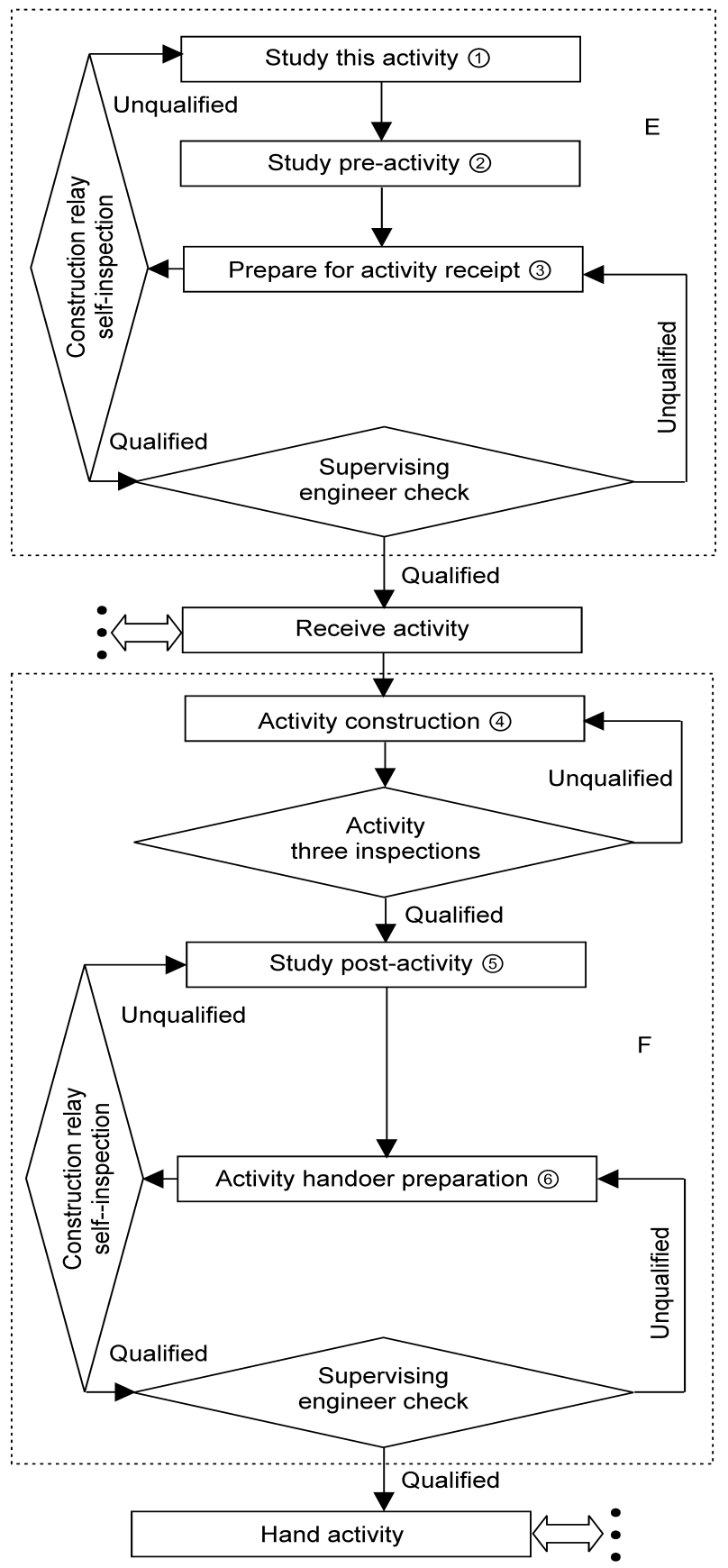

Fig. (4). Single-RCSH flow chart.

corresponding handover speed is calculated and substituted in equations (5), (6), and (7). Following this, the balancing speeds are compared with the activity handover calculation speeds of $d$, and the element $d$ is dropped if it does not conform to the logical relationship, until it meets element $\mathrm{d}$ in a correct logical relationship.

(1) Research was performed on the activity construction difficulty points, activity personnel and equipment configurations, cooperation and resource allocation situations that may exist, and the acceptance conditions that need to be created.
(2) Pre-activity construction characteristics and handover requirements to be met were studied.

(3) The most effective operation method of activity handover for reaching the optimal interconnection state was studied including handover measures when facing temporary, sudden, or uncertain circumstances in the activity handover process.

(4) The task was with high-quality effort and proactive attitude, provided effective measures to ensure effective implementation, and strived to meet expectations for the task. 


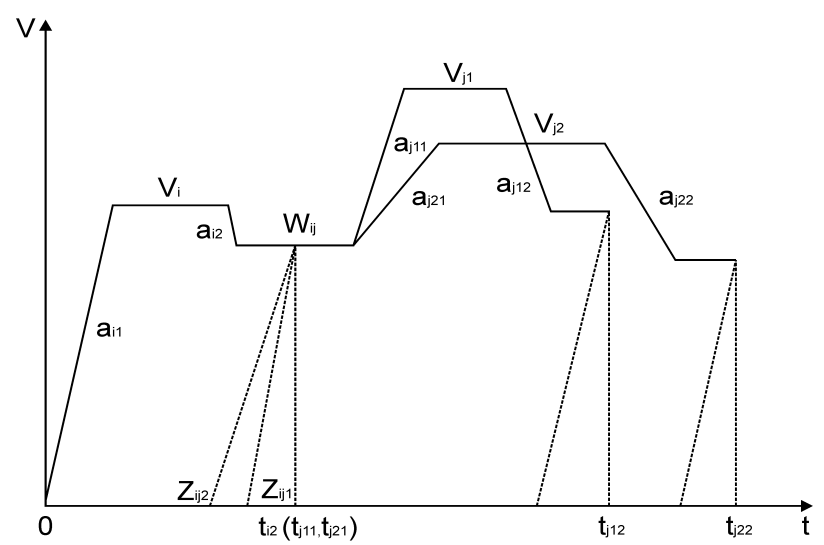

Fig. (5). Schematic diagram of handover between single-activity and multi-activities.

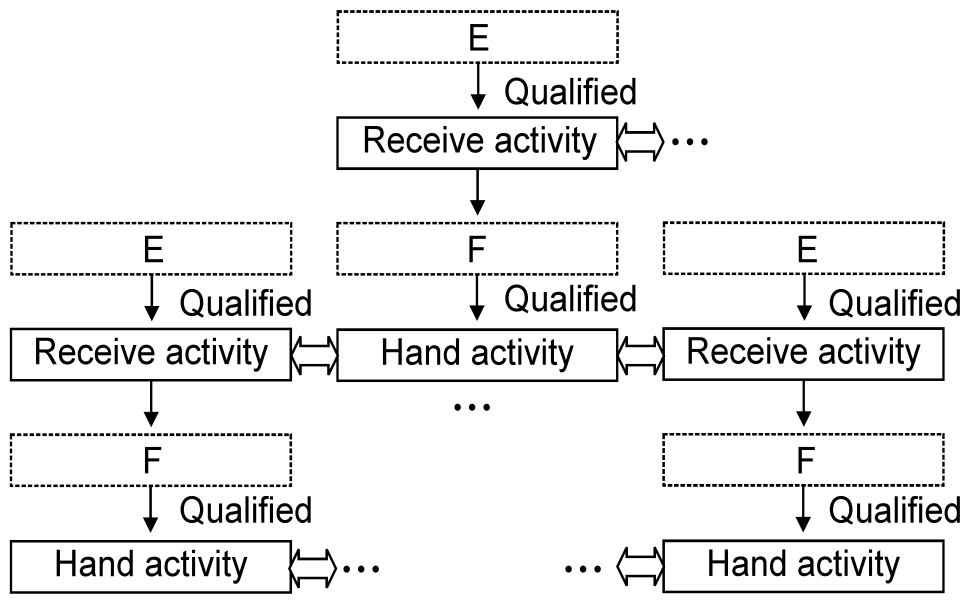

Fig. (6) Flow chart for handover between single-activity and multi-activities.

(5) The post-activity construction characteristics, personnel and equipment configuration situations were studied along with the handover requirements needed to be met.

(6) Adherence to post-activity by the "user" and safely hand it on time with guaranteed quality and quantity. The operational results of the activity which can meet postactivity requirements were carefully verified. Additionally, in accordance with the requirements, a wide range of questions were answered raised during activity handover to reach the optimal interconnection state.

\subsection{Multiple RCSH}

\subsubsection{Single-to-Multiple Activity Handover}

Handover between a single activity and multiple activities refers to a complete handover of a single activity simultaneously to multiple activities. From the relay chain operation principle-the closer the in-activity and ex-activity handover speeds, the higher is the relay efficiency-it is observed that a high handover efficiency can be obtained when the activity handover occurs at the same handover speed.
Schematic diagram of handover between single-activity and multi-activities is shown in Fig. (5). Flow chart for handover between single-activity and multi-activities is shown in Fig. (6).

In the balancing-speed solving process, let the handover calculation speed $\mu \mathrm{ij} 0=$ vi of the relay event comprise a single in-activity and multiple ex-activities, so that the corresponding handover speed is $\omega \mathrm{ij} 0=\beta \mathrm{vi}$. According to the activity, balancing-speed solving methods proposed in 2.3.3, activity $\mathrm{i}$ and jm were calculated as relative balancing speeds in their respective single relay chain, expressed as vi1, vj1, vi2, $\mathrm{vj} 2, \ldots$, vim, vjm, for which: $\mathrm{m}$ is the number of ex-activities. For each activity, balancing speed was calculated according to $\mu \mathrm{ij}=\min \{v i 1, \mathrm{vj} 1, \mathrm{vi} 2, \mathrm{vj} 2, \ldots, \mathrm{vim}, \mathrm{vjm}\}$ and the balancing-speed solving methods in 2.3.3.

\subsubsection{Multiple- To Single-Activity Handover}

Planned durations of in-activities vary; therefore, finish times vary as well. If handover takes place between multiple in-activities and a single ex-activity after all in-activities are implemented, it will not only waste resources but affect 


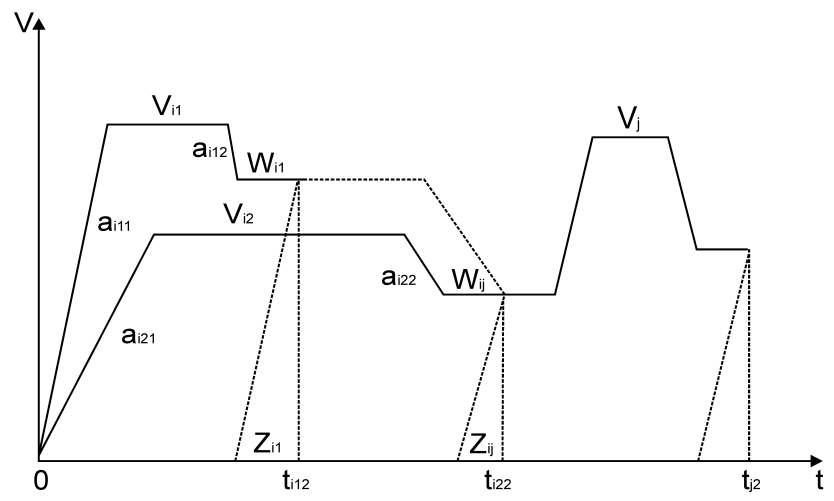

Fig. (7). Schematic diagram of handover between multi-activities and single-activity.

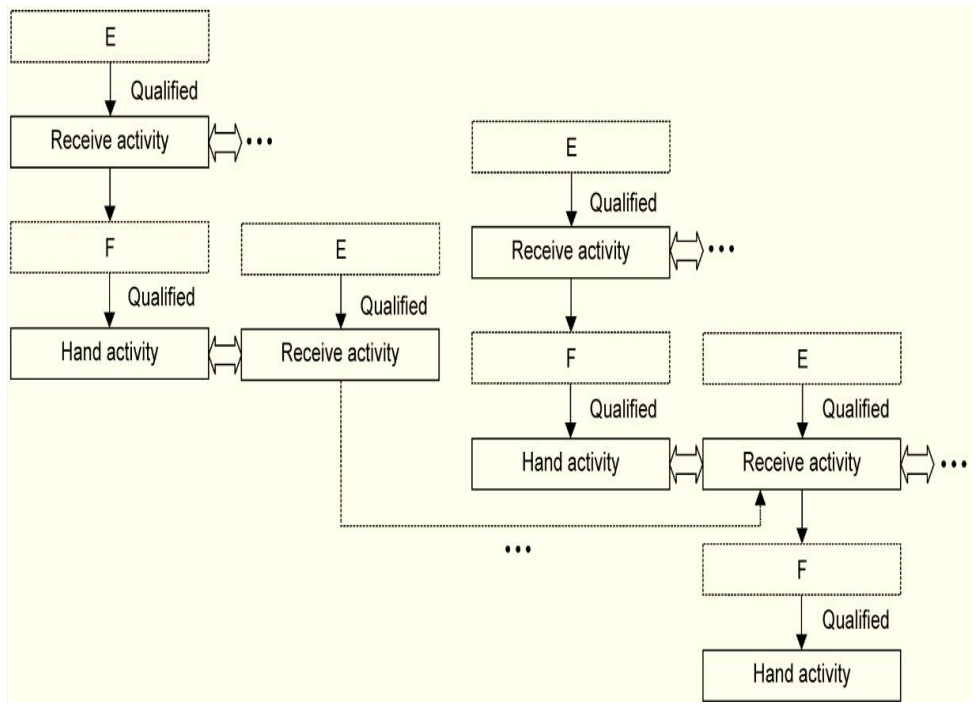

Fig. (8). Flow chart for handover between multi-activities and single-activity.

normal project operation. To ensure timeliness and efficiency of the handover, in-activities (activities with non-maximal planned durations) and single ex-activity should exchange, it was assumed that they moved forward in their respective handover speed after exchanging, and then decelerated to the handover speed $\omega \mathrm{ij}=\beta \mathrm{vij}$ (vij is the minimum balancing speed of the in-activities and ex-activity). The ex-activity accelerated to its balancing speed after handover between inactivities (activities with maximal planned durations) and the ex-activity. Figs. (7 and 8) are a schematic diagram and a flow chart, respectively, of handover between multiple activities and a single activity.

In the balancing-speed solving process, the corresponding planned duration of in-activity was $i 1, i 2, \ldots, i m$ are $T_{i 1}$, $T_{i 2}, \ldots, T_{i m}$, for which: $m$ is the number of in-activities. Take $T_{i k}=\max \left\{T_{i 1}, T_{i 2}, \ldots, T_{i m}\right\}, k=1,2, \ldots, m$. Let $n$ be the number of in-activities with maximal planned duration. $\mu_{i e}=v_{i e}$ was substituted in equation (5) and the corresponding activity balancing speed $v_{i e}, e=1,2, \ldots, k-1, k+1, \ldots, m$ was obtained. The handover calculation speed of the relay event was set where handover occurred between in-activities (with maximal planned duration) and the single ex-activity was $\mu_{i j 0}=v_{j}$, therefore, the corresponding handover speed was $\omega_{i j 0}=\beta v_{j}$. According to the activity balancing-speed solving methods in 2.3.3, the in-activity $i k$ and ex-activity $j$ relative balancing speeds were calculated in their respective single relay chain, expressed as $v_{i k 1}, v_{j l}, v_{i k 2}, v_{j 2}, \ldots, v_{i k n}, v_{j n}$. Balancing speed for each activity was calculated according to $\mu_{i j}=$ $\min \left\{v_{i k 1}, v_{j l}, v_{i k 2}, v_{j 2}, \ldots, v_{i k n}, v_{j n}, v_{i e}\right\}$ through the activity balancing-speed solving methods in 2.3.3.

\subsubsection{Handover Between Multiple Activities}

Handover from multiple activities to multiple activities was decomposed into a multiple in-activities exchange with a single activity $b(\mathrm{~Tb}=0)$, and then a single activity $\mathrm{b}$ exchanged with multiple ex-activities. This breakdown revealed characteristics of a handover between both a single activity and multiple activities, and between multiple activities and a single activity. Schematic diagram of a handover between multiple activities and multiple activities is shown in Fig. (9). Flow chart of the handover between multiple activities and multiple activities is shown in Fig. (10). 


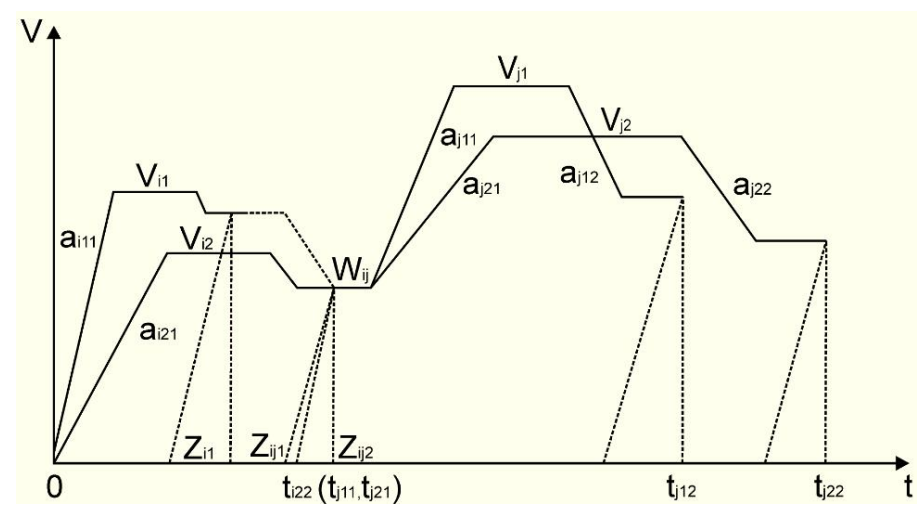

Fig. (9). Schematic diagram of a handover between multiple activities and multiple activities.

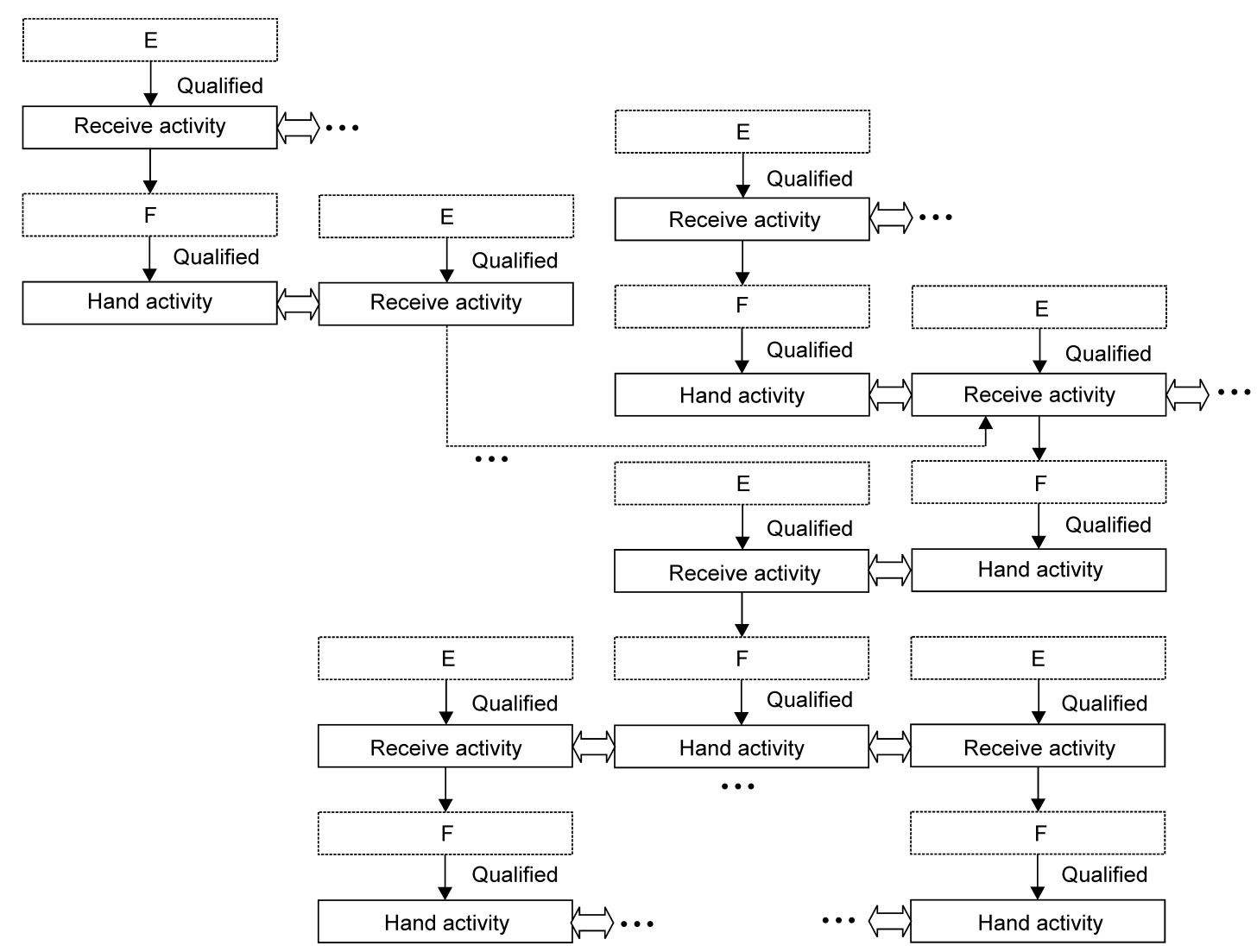

Fig. (10). Flow chart of the handover between multiple activities and multiple activities.

In the balancing speed solving process, the corresponding planned duration of in-activity $i 1, i 2, \ldots, i m$ was $T_{i 1}, T_{i 2}, \ldots$, $T_{i m}$, in which: $m$ is the number of in-activities. Let $T_{i k}=$ $\max \left\{T_{i}, T_{i 2}, \ldots, T_{i m}\right\}$, and $k=1,2, \ldots, m$. Let $n$ be the number of in-activities with maximal planned duration. $\mu_{i e}=v_{i e}$ was substituted in equation (5) and the corresponding activity balancing speed $v_{i e}, e=1,2, \ldots, k-1, k+1, \ldots, m$ was obtained. According to equations (5), (6) and (7), the relative balancing speed of activity $i k$ and $j$ was calculated in their respective single relay chain, expressed as $v_{i k 11}, v_{j 11}, v_{i k 12}, v_{j 21}$, $\ldots, v_{i k l p}, v_{j p l}, v_{i k 21}, v_{j 12}, v_{i k 22}, v_{j 22}, \ldots, v_{i k 2 p}, v_{j p 2}, \ldots, v_{i k n}, v_{j l n}$, $v_{i k n 2}, \quad v_{j 2 n}, \ldots, \quad v_{i k n p}, v_{j p n}$, where: $p$ is the number of ex-activities. Each balancing speed for each activity was calculated according to $\mu_{i j}=\min \left\{v_{i k 11}, v_{j 11}, v_{i k 12}, v_{j 21}, \ldots, v_{i k l p}\right.$, $v_{j p 1}, v_{i k 21}, v_{j 12}, v_{i k 22}, v_{j 22}, \ldots, v_{i k 2 p}, v_{j p 2}, \ldots, v_{i k n}, v_{j l n}, v_{i k n 2}, v_{j 2 n}$, $\left.\ldots, v_{i k n p}, v_{j p n}, v_{i e}\right\}$ through balancing-speed solving methods in 2.3.3.

\section{DISCUSSION}

Using the single relay chain as an example, the characteristics of RCSH were discussed in comparison to traditional activity handover. In addition, using the multiple relay chain as an example, the same characteristics as RCSH were obtained. 
$I, j, k$ represent interconnected activities in a sequence. Let activity $i$ : staffing 10 persons (engineer 1 person, assistant engineer 2 persons, mechanic 7 persons), planned duration $T_{i}=6$, project quantity was $A_{i}=60$, with difficulty degree $1 / B_{i}=0.95$, equipment configuration and utilization ratio, respectively, were $P_{i}=0.99, S_{i}=0.95$, resources reserve coefficients were $\lambda_{\mathrm{i}}=\lambda_{\mathrm{j}}=\lambda_{\mathrm{k}}=1.2$, and acceleration was $a_{i 1}=3, a_{i 2}=-3$. Let activity $j$ : staffing 15 persons (engineer 1 person, assistant engineer 3 persons, mechanic 11 persons), planned duration $T_{j}=8$, project quantity was $A_{j}=$ 120 , with difficulty degree $1 / B_{j}=0.9$, equipment configuration and utilization ratio, respectively, were $P_{j}=0.98, S_{j}=$ 0.95 , and acceleration was $a_{j 1}=2, a_{j 2}=-2$. Let activity $k$ : staffing 12 persons (engineer 1 person, assistant engineer 2 persons, mechanic 9 persons), planned duration $T_{k}=6$, project quantity was $A_{k}=72$, with difficulty degree $1 / B_{k}=0.95$, equipment configuration and utilization ratio, respectively, were $P_{k}=0.98, S_{k}=0.95$, and acceleration was $a_{j 1}=2, a_{j 2}=$ 2.

\subsection{Balancing-Speed Calculation Under Varying Condi- tions}

Let the traditional handover time of construction activity $i$ and $j$ be $t_{i j}=0.5$. Substituting the given data into Eqs. (3) and (4), balancing speeds of activity $j$ under conditions of the traditional handover and single-RCSH (static) were obtained, expressed as $v_{j 0}=0.98, v_{j 1}=0.91$. Letting the handover speed conversion coefficient be $\beta=0.9$, the solving process of balancing speed $v_{j 2}$ was expressed as:

$M=\left\{d_{1}=\left\{\mu_{i j}=v_{j 2}, \quad \mu_{j k}=v_{j 2}\right\}, \quad d_{2}=\left\{\mu_{i j}=v_{i 2}, \quad \mu_{j k}=v_{k 2}\right\}\right.$, $\left.d_{3}=\left\{\mu_{i j}=v_{j 2}, \mu_{j k}=v_{k 2}\right\}, d_{4}=\left\{\mu_{i j}=v_{i 2}, \mu_{j k}=v_{j 2}\right\}\right\}$, when taking $d_{1}=$ $\left\{\mu_{i j}=v_{j 2}, \mu_{j k}=v_{j 2}\right\}$, substituting $\omega_{i j}=\beta v_{j 2}, \omega_{j k}=\beta v_{j 2}$ into Eqs. (5), (6), and (7), we get (to conveniently calculate, let $\omega_{k l}=0$; the calculation method is the same when $\omega_{k l} \neq 0$ ):

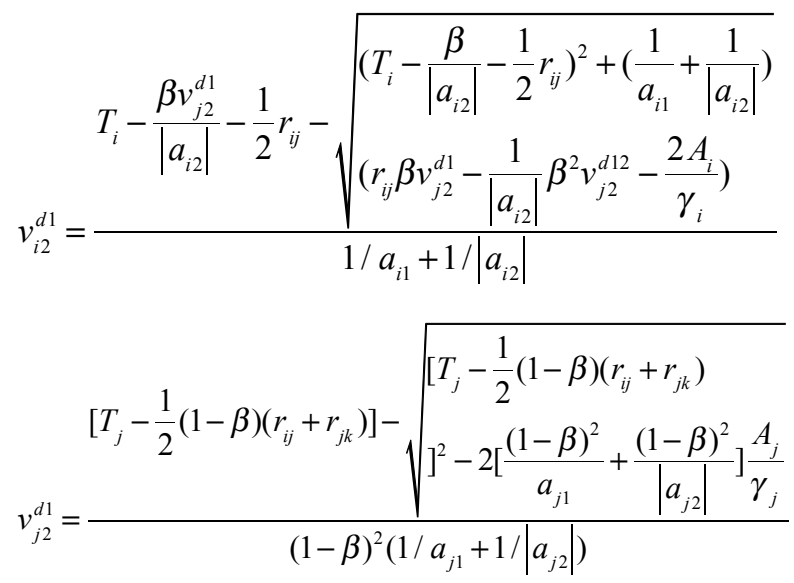

$$
v_{k 2}^{d 1}=\frac{T_{k}-\frac{1}{2} r_{j k}+\frac{\beta v_{j 2}^{d 1}}{a_{k 1}}-\sqrt{\left(\frac{1}{a_{k 1}}+\frac{1}{\left|a_{k 2}\right|}\right)\left(\beta r_{j k} v_{j 2}^{d 1}-\frac{\beta^{2} v_{j 2}^{d 12}}{a_{k 1}}-\frac{2 A_{k}}{\gamma_{k}}\right)}}{1 / a_{k 1}+1 /\left|a_{k 2}\right|}
$$

Substituting the given data into Eqs. (8), (9), and (10), $v_{i 2}{ }^{d l}=0.84, v_{j 2}{ }^{d 1}=0.862$, and $v_{k 2}{ }^{d 1}=0.844$ was obtained, therefore, $v_{i 2}{ }^{d l}<v_{j 2}{ }^{d l}$ and $v_{k 2}{ }^{d l}<v_{j 2}{ }^{d l}$, this contradicts with $d_{1}=$ $\left\{\mu_{i j}=v_{j 2}, \mu_{j k}=v_{j 2}\right\}$, and was dropped. Taking $d_{2}=\left\{\mu_{i j}=v_{i 2}\right.$, $\left.\mu_{j k}=v_{k 2}\right\}$, substituting $\omega_{i j}=\beta v_{i 2}, \omega_{j k}=\beta v_{k 2}$ into Eqs. (5), (6), and (7), we get:

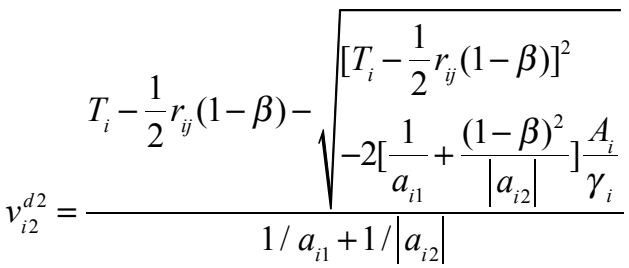

$$
\begin{aligned}
& v_{k 2}^{d 2}=\frac{T_{k}-\frac{1}{2} r_{j k}(1-\beta)-\sqrt{\left[T_{k}-\frac{1}{2} r_{j k}(1-\beta)\right.}}{(1-\beta)^{2} / a_{k 1}+1 /\left|a_{k 2}\right|} \\
& v_{j 2}^{d 2}=\frac{T_{j}-\frac{1}{2} r_{i j}-\frac{1}{2} r_{j k}+\frac{\beta}{a_{j 1}} v_{i 2}^{d 2}+\frac{\beta}{\left|a_{j 2}\right|} v_{k 2}^{d 2}}{1 / a_{j 1}+1 /\left|a_{j 2}\right|}-
\end{aligned}
$$

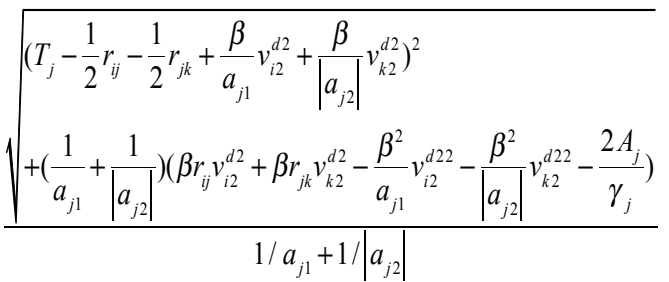

Substituting the given data into Eqs. (11), (12), and (13), vi $2 \mathrm{~d} 2=0.77, \mathrm{vj} 2 \mathrm{~d} 2=0.864$, and $\mathrm{vk} 2 \mathrm{~d} 2=0.85$ were obtained, therefore $v i 2 \mathrm{~d} 2<\mathrm{vj} 2 \mathrm{~d} 2$ and $\mathrm{vk} 2 \mathrm{~d} 2<\mathrm{vj} 2 \mathrm{~d} 2$, was consistent with the logical relationship of $\mathrm{d} 1=\{\mu \mathrm{ij}=\mathrm{vj} 2, \mu \mathrm{jk}=\mathrm{vj} 2\}$. Similarly, it was verified that $\mathrm{d} 3$ and $\mathrm{d} 4 \mathrm{did}$ not conform and were dropped.

\subsection{Balancing Speed Ratio Curve: Single-RCSH (Static) To Traditional Handover}

The curve of the balancing speed ratio of single-RCSH (static) to traditional handover between single activities was outlined with handover time. Let balancing speed ratio of single-RCSH (static) to traditional handover between single activities be $\eta_{j l}$, expressed as $\eta_{j l}=v_{j l} / v_{j 0}$, with handover time as $t_{i j}$. According to Eqs. (3) and (4), we get:

$$
\eta_{j 1}=\frac{v_{j 1}}{v_{j 0}}=\frac{T_{j}-\sqrt{T_{j}^{2}-\left(\frac{1}{a_{j 1}}+\frac{1}{\left|a_{j 2}\right|}\right) \frac{2 A_{j}}{\gamma_{j}}}}{T_{j}-t_{i j}-\sqrt{\left(T_{j}-t_{i j}\right)^{2}-\left(\frac{1}{a_{j 1}}+\frac{1}{\left|a_{j 2}\right|}\right) \frac{2 A_{j}}{\gamma_{j}}}}
$$

Substituting the given data into Eq. (14), the relation curve of $\eta_{j l}$ with $t_{i j}$ is shown in Fig. (11). 


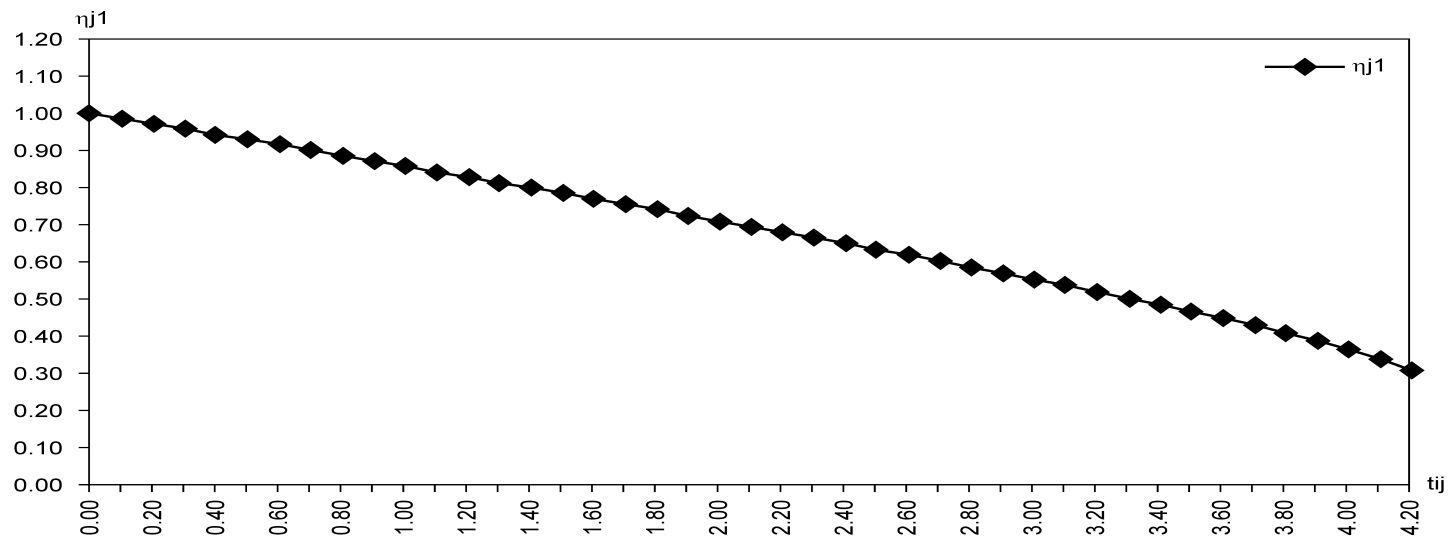

Fig. (11). Relation curve of $H_{J I}$ with $T_{i j}$.

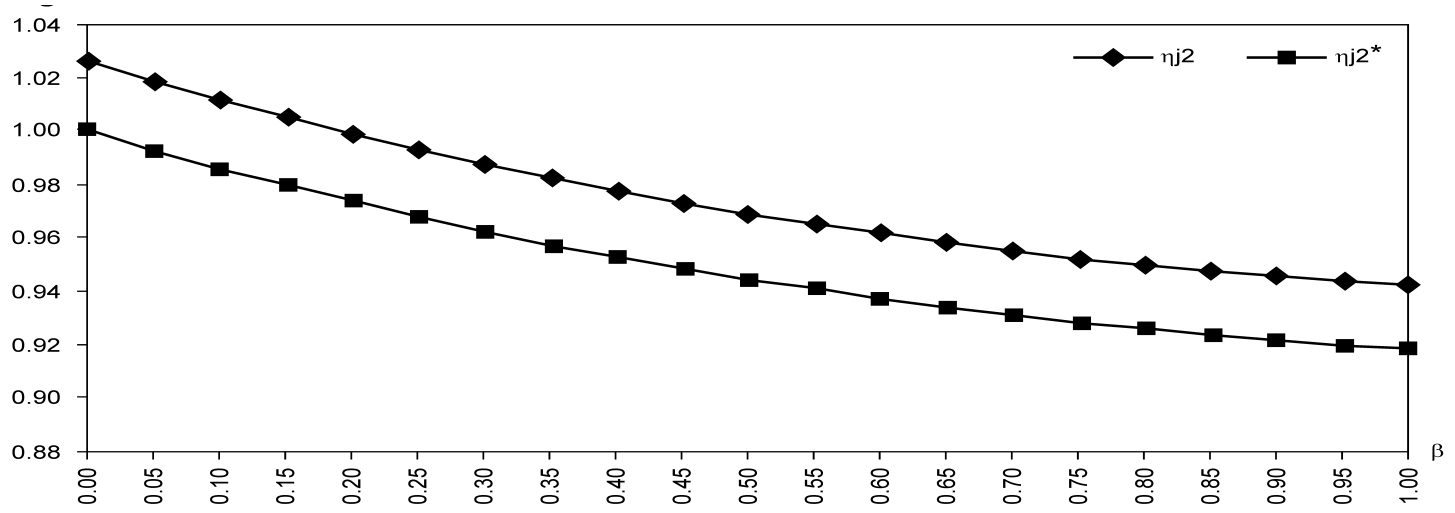

Fig. (12). Relation curve of $\mathrm{HJ} 2$ with B.

\subsection{Balancing Speed Ratio Curve: Single-RCSH To Sin- gle-RCSH (Static) With B}

Let the balancing speed ratio of single-RCSH to singleRCSH (static) be $\eta_{j 2}$, expressed as $\eta_{j 2}=v_{j 2} / v_{j 1}$. According to Eqs. (4) and (13), we get:

$$
\begin{aligned}
\eta_{j 2}= & \frac{v_{j 2}}{v_{j 1}}=\frac{v_{j 2}^{d 2}}{v_{j 1}}=\frac{T_{j}-\frac{1}{2} r_{i j}-\frac{1}{2} r_{j k}+\frac{\beta}{a_{j 1}} v_{i 2}^{d 2}+\frac{\beta}{\left|a_{j 2}\right|} v_{k 2}^{d 2}}{T_{j}-\sqrt{T_{j}^{2}-\left(\frac{1}{a_{j 1}}+\frac{1}{\left|a_{j 2}\right|}\right) \frac{2 A_{j}}{\lambda_{j}}}}- \\
& \sqrt{\frac{\left(T_{j}-\frac{1}{2} r_{i j}-\frac{1}{2} r_{j k}+\frac{\beta}{a_{j 1}} v_{i 2}^{d 2}+\frac{\beta}{\left|a_{j 2}\right|} v_{k 2}^{d 2}\right)^{2}+\left(\frac{1}{a_{j 1}}\right)\left(\beta r_{i j} v_{i 2}^{d 2}+\beta r_{j k} v_{k 2}^{d 2}-\frac{\beta^{2}}{a_{j 1}} v_{i 2}^{d 22}-\frac{\beta^{2}}{\left|a_{j 2}\right|} v_{k 2}^{d 22}-\frac{2 A_{j}}{\gamma_{j}}\right)}{T_{j}-\sqrt{T_{j}^{2}-\left(\frac{1}{a_{j 1}}+\frac{1}{\left|a_{j 2}\right|}\right) \frac{2 A_{j}}{\lambda_{j}}}}}
\end{aligned}
$$

Substituting Eqs. (11) and (12) into Eq. (15), and the given data into Eq. (15), the relation curve of $\eta_{j 2}$ with $\beta$ is shown in Fig. (12) $\left(\eta_{j 2}{ }^{*}\right.$ is the corrective value of $\eta_{j 2}$, deviating from the calculation).
3.4. Balancing Speed Ratio Curve: Single RCSH To Traditional Handover With $T_{i j}$ and $B$

$$
\begin{aligned}
& \eta_{j 3}= \frac{v_{j 2}}{v_{j 0}}=\frac{v_{j 2}^{d 2}}{v_{j 0}}=\frac{T_{j}-\frac{1}{2} r_{i j}-\frac{1}{2} r_{j k}+\frac{\beta}{a_{j 1}} v_{i 2}^{d 2}+\frac{\beta}{\left|a_{j 2}\right|} v_{k 2}^{d 2}}{T_{j}-t_{i j}-\sqrt{\left(T_{j}-t_{i j}\right)^{2}-\left(\frac{1}{a_{j 1}}+\frac{1}{\left|a_{j 2}\right|}\right) \frac{2 A_{j}}{\lambda_{j}}}}- \\
& \sqrt{\left(T_{j}-\frac{1}{2} r_{i j}-\frac{1}{2} r_{j k}+\frac{\beta}{a_{j 1}} v_{i 2}^{d 2}+\frac{\beta}{\left|a_{j 2}\right|} v_{k 2}^{d 2}\right)^{2}} \\
&+\left(\frac{1}{a_{j 1}}+\frac{1}{\left|a_{j 2}\right|}\right)\left(\beta r_{i j} v_{i 2}^{d 2}+\beta r_{j k} v_{k 2}^{d 2}-\frac{\beta^{2}}{a_{j 1}} v_{i 2}^{d 22}-\frac{\beta^{2}}{\left|a_{j 2}\right|} v_{k 2}^{d 22}-\frac{2 A_{j}}{\gamma_{j}}\right) \\
& T_{j}-t_{i j}-\sqrt{\left(T_{j}-t_{i j}\right)^{2}-\left(\frac{1}{a_{j 1}}+\frac{1}{\left|a_{j 2}\right|}\right) \frac{2 A_{j}}{\lambda_{j}}}
\end{aligned}
$$

Substituting Eq. (11) and (12) into Eq. (16), and the given data into Eq. (16), the relation curve of $\eta \mathrm{j} 3$ with tij and $\beta$ is shown in Fig. (13).

\subsection{Comparison And Analysis}

The balancing-speed relationship among traditional handover, $\mathrm{RCSH}$ (static), and $\mathrm{RCSH}$ was $v_{j 2} \leq v_{j 1} \leq v_{j 0}$. As 


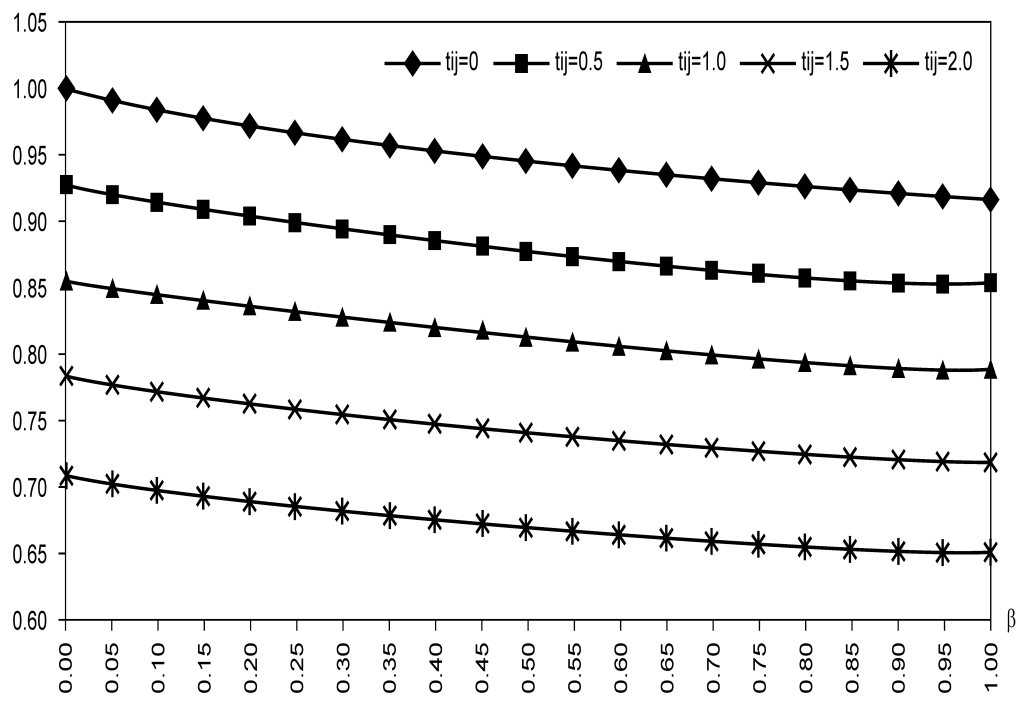

Fig. (13). Relation curve of $\mathrm{Hj} 3$ with Tij and B.

certified under different handover conditions, the total project quantities remained unchanged and staffing had a fixed value, so that $V T=A / \gamma$ was fixed. The v-t graph shows that the area surrounded by the activity speed curve and t-axis was unchanged. According to Figs. (1 and 2), $v_{j 2} \leq v_{j l} \leq v_{j 0}$ can be obtained if and only if $t_{i j}=0, \beta=0$, an equal sign can be obtained. According to the instance calculation results $v_{j 0}=$ $0.98, v_{j 1}=0.91, v_{j 2}=0.864, \quad v_{j 2}<v_{j 1}<v_{j 0}$, was obtained which shows that RCSH had minimum balancing speed and construction intensity, thus ensuring the maximum completion probability for engineering activity, the highest construction quality level, and the greatest risk-resistance ability.

The balancing speed ratio of RCSH (static) to traditional handover decreased with increase in traditional activity handover time; suggesting that, the longer the handover time, the greater is the traditional activity balancing speed, the higher is the construction intensity, the smaller is the probability of timely completion, and the smaller is the riskresistance ability.

The balancing speed ratio of RCSH to RCSH (static) decreased with increase in $\beta$, suggesting that the greater the handover speed of RCSH, the smaller is the balancing speed, the greater is the probability of timely completion, the higher is the construction quality level, and the greater is the riskresistance ability.

The balancing speed ratio of RCSH to traditional handover decreased with increase in handover time $t(\beta$ unchanged), and decreased with increase in $\beta$ ( $t$ unchanged). In the third phase of the Three Gorges Dam Project, $\beta$ reached 0.9 ; if $t_{i j}=0.5, \eta_{j 3}$ can reach 0.85 ; that is activity handover can be conducted at a rapid speed, which ensures that the relative speed is sufficiently small for smooth exchange of activities. On the other hand, the construction balancing speed was relatively small, which reached 0.85 of the traditional handover speed, bringing about a significant change and contribution to the project's quality and duration.

\section{CONCLUSION}

The technology of engineering projects is complex and the projects themselves involve many processes. In construction management, even minor delays in activity handover can cause serious consequences, including total duration delay, enormous waste of resources, exorbitant costs, and so on. Therefore, in engineering quality and schedule control, the proposed RCSH technology has great significance and advantages over traditional activity handover.

RCSH technology effectively solves the problems of mutual cooperation and coordination in the production process. It eliminates lack of drive at the beginning of the activity and the subsequent need to compensate towards the end of the engineering activity; thereby ensuring production balance. It also places significant emphasis on activity interconnecting links. Operation links are typically conducted in a created environment; on the other hand, interconnecting links are associated with the creation of external conditions, while enabling active team collaboration and cohesion.

Through its resource reserve and release, RCSH technology has also advanced the elastic adaptation to various types of unknown variables, from unformed operations to severe environments. RCSH technology further defines responsibilities between departments and activities, forms a type of job chain belt, fosters subjective initiatives and creativity of staff, raises awareness of timeliness, and ensures engineering quality and duration.

By upholding the activity handover ("relay") event as key and by focusing on the study of activity receipt, RCSH technology can effectively address the significant legacy of issues that have arisen from a primary focus on the "handing off" while ignoring the receiving component. In terms of total quality management, RCSH technology enriches and develops existing program management methods, while innovating aspects of modern management theory. As a new management method for non-linear operation linking, RCSH 
technology has been applied in the Three Gorges Dam construction project, where it has already achieved success. One of the method's most significant achievements was in the generalization and implementation of the third phase of the Three Gorges Dam Project's concrete construction quality control process, which effectively created the construction miracle of a concrete dam without a crack. By further generalizing and applying this method to engineering construction practices-consistently evaluating post-project experiences and continually refining the method-will continue producing significant achievements and economic benefits in future engineering construction.

\section{CONFLICT OF INTERESTS}

The authors declare that there is no conflict of interests regarding the publication of this article.

\section{ACKNOWLEDGEMENTS}

Declared none.

\section{REFERENCES}

[1] J. Nedbalek, "New type of RBF network in graph applications," International Journal of Performability Engineering, vol. 8, no. 5, pp. 481-488, 2012.

[2] Z. Li, and L. Zhang, "A Study on multi-project management based on gantt chart and network planning technique," Applied Mechanics and Materials, vol. 174, pp. 2854-2860, 2012.

[3] A. Sadegheih, "Optimization of network planning by the novel hybrid algorithms of intelligent optimization techniques," Energy, vol. 34, no. 10, pp. 1539-1551, 2009.

[4] L. Hu, and D. Zhong, "Research on network scheduling planning technique based on system simulation," Proceedings of the 2010 IEEE International Conference on Progress in Informatics and Computing, pp. 1084-1087, 2010.

[5] S. Biffl, B. Thurnher, Goluch, G., D. Winkler, W. Aigner, and S. Miksch, "An empirical investigation on the visualization of temporal uncertainties in software engineering project planning," International Symp. Empirical Software Engineering, pp. 437-446, 2005.
[6] M. Tory, S. Staub-French, D. Huang, Y.L. Changc, and C. Swindellsa, "Rachel pottinger comparative visualization of construction schedules," Automation in Construction, vol. 29, no. 1, pp.68-82, 2013.

[7] Latour, "Mixing humans and nonhumans together: the sociology of a door-closer," Social Problem, vol. 35, no. 3, pp. 298-310, 1988.

[8] Latour. "Pragmatogonies," American Behavioural Scientist, vol. 37, no. 6, pp. 791-808, 1994 .

[9] B. Latour, "For david blood and beyond: a reply to david bloor anti-latour," Studies in the History and Philosophy of Science, vol. 85 , no. 30, pp. 114-115, 1999.

[10] J. Law, "Notes on the theory of actor-network: ordering, strategy and heterogeneity," Systemic Practice and Action Research, vol.5, no. 4, pp. 379-393, 1992.

[11] L. Lindkvist, "Project organization: exploring its adaptation properties," International Journal of Project Management, vol. 26, no. 1, pp. 113-120, 2008.

[12] M. Lindahl, and A. Rehn, "Towards a theory of project failure." International Journal of Management Concepts and Philosophy, vol. 2, no. 3, pp. 246-254, 2007.

[13] A. Sidorova, and S. Sarker, "Unearthing some causes of BPR failure: An actor-network theory perspective," Journal of Management Information Systems, vol. 1, no. 119-132, 2002.

[14] S. Daniel, D. Andrew, and B. Naomi, "How actor-network theories can help in understanding project complexities," International Journal of Managing Projects in Business, vol. 4, no. 2, pp. 274293, 2011.

[15] A. P. C. Chan, D. Scott, and A. P. L. Chan, " Factors affecting the success of a construction project,"Journal of Construction Engineering and Management, vol. 130, no. 1, pp. 153-155, 2004.

[16] S. Blackburn, "The project manager and the project-network," International Journal of Project Management, no. 20, pp. 199-204, 2002.

[17] Sage, A. Dainty, and N. Brookes, "Who reads the project file? Exploring the power effects of knowledge tools in construction management research,"Construction Management and Economics, vol. 28, no. 6, pp. 629-640, 2010.

[18] K. Tryggestad, S. Georg, and T. Hernes, "Construction buildings and design ambitions," Construction Management and Economics, vol. 28 , no. 6, pp. 695-706, 2010.

[19] A. C .A. Cauvin, A. F. A. Ferrarini, and E. T. E. Tranvouez, "Disruption management in distributed enterprise: A multi-agent modeling and simulation of cooperative recovery behaviours," International Journal of Production Economics, vol. 122, no. 6, pp.429439, 2009.

\footnotetext{
Received: July 05, 2015

Revised: August 15, 2015

Accepted: September 11, 2015

(C) Wang et al.; Licensee Bentham Open.
}

This is an open access article licensed under the terms of the Creative Commons Attribution Non-Commercial License (http://creativecommons.org/licenses/by$\mathrm{nc} / 4.0 /$ ) which permits unrestricted, non-commercial use, distribution and reproduction in any medium, provided the work is properly cited. 